Forum $2017 \cdot 32: 266$

DOI 10.1007/s12312-017-0256-4

Online publiziert: 19. April 2017

○) Springer Medizin Verlag GmbH 2017

KREBSVERBAND

:: BADEN-WÜRTTEMBERG E.V.

Prof. Albers, Präsident der Deutschen Krebsgesellschaft, hat im Forum 6/2016 eindeutig Position bezogen: „In Deutschland leben mittlerweile geschätzt 1,5 Millionen Menschen mit Krebs. Fast die Hälfte aller Krebspatienten erkranken im erwerbsfähigen Alter; über ein Drittel derer, die vor der Erkrankung gearbeitet haben, findet nicht mehr zurück ins Erwerbsleben oder sieht sich mit hohen Erwartungen an seine Leistungsfähigkeit konfrontiert, die er nicht immer erfüllen kann. Hier ist vor allem eine gute Beratung gefragt“. Er forderte zu Recht die Sicherung der gesellschaftlichen und beruflichen Teilhabe von Menschen mit einer Erkrankung. „Die ambulanten Psychosozialen Krebsberatungsstellen der Landeskrebsgesellschaften leisten in diesem $\mathrm{Zu}$ sammenhang hervorragende Arbeit für Krebspatienten und deren Angehörige“.

Ich habe im Folgeheft diese eindeutige Positionierung von Herrn Prof. Albers nachdrücklich begrüßt und auf eine hoff-

Krebsverband Baden-Württemberg e.V., Stuttgart

\title{
Finanzierung ambulanter Krebsberatungsstellen
}

\section{Bemerkenswertes ist in Baden-Württemberg gelungen}

nungsvolle Entwicklung in Baden-Württemberg hingewiesen. Dort hatte nicht nur das Land Baden-Württemberg 2016 für den Aufbau weiterer bedarfsgerechter Krebsberatungs-stellen - neben den DKH modellfinanzierten Krebsberatungsstellen - 1,2 Millionen zur Verfügung gestellt. Für die Folgefinanzierung der im Landesprojekt geförderten Beratungsstellen gelang nunmehr eine beispielgebende Finanzierungsvereinbarung: Das Land Baden-Württemberg, die gesetzlichen Krankenversicherung, die gesetzliche Rentenversicherung, Kommunen, gemeinnützige Organisationen, der Krebsverband Baden-Württemberg und die Wirtschaft sichern gemeinsam die Finanzierung auch für das Jahr 2017!

Damit wird einerseits ein deutliches Signal gesetzt, dass es sich bei der psychosozialen Beratung krebskranker Menschen um eine Gemeinschaftsaufgabe aller relevanten gesellschaftlichen Insti- tutionen handelt. Insbesondere die privaten Krankenversicherungen werden sich dieser Verantwortung auf Dauer nicht entziehen können. Klar wird damit aber auch, dass nunmehr der Bundesgesetzgeber für eine notwendige Regelfinanzierung ab 2018 in besonderer Verantwortung steht!

Die Presserklärung des Sozialministeriums Baden-Württemberg vom 4. Februar 2017 ist zu finden unter https://sozialministerium.baden-wuerttemberg.de/ de/service/presse/pressemitteilung/pid/ lucha-kaempft-fuer-dauerhaften-fortbestand-der-krebsberatungsstellen/

\section{Korrespondenzadresse}

\section{H. Seiter}

Krebsverband Baden-Württemberg e.V. Adalbert-Stifter Straße 105, 70437 Stuttgart Tel: 0711 848-10770

E-Mail: info@krebsverband-bw.de Internet: www.krebsverband-bw.de 